\title{
Toma de decisión para colocar mantenedor de espacio después de la pérdida prematura de primeros molares primarios: Revisión de literatura
}

Elizabeth Fiorella Pino Guerrero';

Jorge Luis Castillo Cevallos';

\section{Resumen}

Introducción: La pérdida prematura de molares primarios ha sido asociada con la perdida de espacio y dificultades eruptivas. Sin embargo, existen opiniones diferentes sobre la necesidad de colocar mantenedor de espacio después de la pérdida prematura de un primer molar primario. El propósito de esta revisión es proporcionar un resumen de la evidencia disponible respecto a la colocación de mantenedor de espacio después de la perdida prematura del primer molar primario.

Métodos: Se realizó una revisión de literatura en base de datos electrónicas desde el año 1977 hasta el 2016, en las bases de datos (Pubmed, Medline, EBSCO).

Resultados: La mayor parte de estudios concluyeron que la mayor pérdida de espacio ocurre después de la perdida prematura de un primer molar primario mandibular. Muchos factores condicionan está perdida, como lo son la falta de espacio disponible, el status de erupción del primer molar permanente, el momento de la perdida prematura, entre otros.

Conclusiones: Según los estudios consultados la magnitud de la pérdida de espacio después de la pérdida prematura de un primer molar primario es clínicamente cuestionable, no tiene un gran efecto en los arcos dentales. Sin embargo se ha demostrado un gran efecto en el sitio de extracción por lo cual el mantenedor de espacio debe ser usado lo más temprano posible para evitar el desplazamiento de los dientes adyacentes. Cabe resaltar que el tamaño de las muestras y la metodología de algunos artículos son limitadas, por lo cual se requiere estudios de mejor calidad metodológica.

Palabras clave: diente primario, arco dental, extracción dental, aparatos ortodóncicos, maloclusión.

\section{Artigo de Revisão}

\section{Tomada de decisão para colocar mantenedor de espaço após da perda prematura de primeiros molares decíduos: Revisão da literatura}

\section{Resumo}

Introdução: A perda prematura de molares decíduos tem sido associada com a perda de espaço e dificuldades eruptivas. Porém, Há opiniões di- ferentes sobre a necessidade de colocar mantenedor de espaço após da perda prematura de um primeiro molar decíduo. O objetivo desta revisão é fornecer um resumo das evidências disponíveis a respeito à colocação de mantenedor de espaço 
após da perda prematura de primeiro molar decíduo.

Métodos: A revisão da literatura foi realizada em bases de dados electrónicas desde ano 1977 hasta 2016, em as bases de dados (PubMed, Medline, EBSCO).

Resultados: A maioria dos estudos concluíram que a maior perda de espaço ocorre após da perda prematura de um primeiro molar decíduo mandíbular. Muitos factores influenciam esta perda, tais como a falta de espaço disponível, o estado de erupção do primeiro molar permanente, o momento da perda prematura, entre outros. Conclusões: De acordo com os estudos consulta- dos a magnitude da perda de espaço após da perda prematura um primeiro molar decíduo é clinicamente questionável, não tem um grande efeito sobre as arcadas dentárias. Porém tem mostrado grande efeito sobre o local de extracção de modo que o mantenedor de espaço deve ser utilizado o mais cedo possível para evitar o deslocamento dos dentes adjacentes. Notavelmente tamanhos de amostra e metodologia de alguns artigo são limitados, portanto, é necessário estudos de melhor qualidade metodológica.

Palavras chave: dente decíduo, arco dental, extração dentária, aparelhos ortodônticos, má oclusão.

\section{Review Article}

\section{Decision making on space maintainer placement after prema- ture loss primary first molars: Literature review}

\section{Abstract}

Introduction: The use of space maintainers after the premature loss of primary molars has been associated with space loss and eruptive difficulties. However, therearedifferentopinionsabout theneed to place space maintainers after the premature loss of a primary first molar. The purpose of this review is to provide a summary of the available evidence regarding whether to place space maintainer after premature loss of the primary first molar.

Methods: A literature review was conducted in electronic databases from 1977 to 2016, in the databases (Pubmed, Medline, EBSCO).

Results: Most studies concluded that the greatest space loss occurs after the premature loss of a primary mandibular first molar. Many factors condition this lost, such as the lack of available space, the eruption status of the first permanent molar, the time of premature loss, among others.
Conclusions: According to the studies consulted the magnitude of loss of space after the premature loss of a primary first molar is clinically questionable, it does not have a large effect on dental arches. However, a great effect has been demonstrated at the extraction site whereby the space maintainer should be used as early as possible to avoid displacement of the adjacent teeth. It should be noted that the sample size and the methodology of some articles are limited, which requires studies of better methodological quality.

Keywords: tooth deciduous, dental arch, tooth extraction, orthodontic appliances, malocclusion.

\section{Introducción}

La dentición primaria va a mantener la oclusión y el espacio necesario para la erupción de los sucesores permanentes. Por ello, es importante 
para el paciente pediátrico que, ante la pérdida prematura de un diente primario, el odontopediatra mantenga la longitud del arco dental antes de la erupción de los dientes permanentes. La exfoliación de los dientes primarios y erupción de los sucesores permanentes es un proceso fisiológico normal; cuando este proceso es interrumpido debido a la extracción prematura de los dientes primarios, una serie de cambios se observarán en los arcos dentales. ${ }^{1}$ La caries dental y los traumatismos son las causas más comunes en la pérdida de dientes, sin embargo, la caries continúa siendo el principal motivo de la alta tasa de pérdida dental prematura. ${ }^{2}$

La pérdida prematura de los molares primarios da lugar a problemas en el desarrollo del arco, reduciendo la longitud necesaria de este para la erupción de los dientes permanentes, debido al desplazamiento mesial durante la erupción de los dientes posteriores y por lo tanto, predispone el apiñamiento, inclinaciones, rotaciones, erupción ectópica y la retención de los dientes permanentes, así como a una erupción prematura de estos. ${ }^{1,3}$

Esta pérdida prematura de dientes puede aumentar la necesidad de tratamiento de ortodoncia, por lo que es muy importante intervenir en casos de extracción o exfoliación prematura. ${ }^{4} \mathrm{El}$ uso de los mantenedores de espacio puede contrarrestar los efectos de esta pérdida y reducir la gravedad de los resultados negativos. ${ }^{5}$ Existe una diferencia en la pérdida de espacio dependiendo el momento de la perdida prematura y el tipo de diente perdido en los niños con dentición mixta. ${ }^{6,7}$

La prevalencia de la pérdida temprana es mayor en los primeros molares cuando se compara con los segundos molares primarios. ${ }^{8}$ No hay mucha controversia en cuanto a la necesidad de colocar un mantenedor de espacio después de perder prematuramente un segundo molar, ya que se producen grandes cambios en el arco dental, sin embargo, hay puntos de vista conflictivos asociados con la necesidad de mantener el espacio después de perder el primer molar primario. Aunque la mayoría de estudios han informado de que la pérdida de espacio casi siempre se produce en el sitio de extracción cuando un primer molar primario se pierde antes de tiempo, la magnitud de la pérdida y el manejo clínico requerido son controversiales. ${ }^{7}$ 9,10 Este artículo tiene como propósito proporcionar un resumen de la evidencia disponible sobre los cambios en el arco dental y la necesidad de colocar un mantenedor de espacio después de haber pedido prematuramente un primer molar primario.

\section{Materiales y métodos:}

Se realizó una revisión de literatura en varias bases de datos electrónicas como PubeMed, MEDLINE, EBSCO, LILACS, EMBASE; desde el año 1977 hasta el 2016. Los términos utilizados en esta búsqueda de la literatura fueron "premature loss", "space mainteiner", "space loss", "tooth deciduous", "dental arch", "tooth extraction".

Después de la búsqueda se encontraron 100 artículos, de los cuales solo 15 cumplieron con los criterios de inclusión.

Para esta revisión de literatura los criterios de inclusión fueron: estudios clínicos longitudinales, ensayos clínicos en humanos en los cuales se haya perdido un primer molar primario, y se excluyeron los reportes de caso y las guías clínicas. 


\section{Resultados:}

Un total de 15 artículos cumplieron con los criterios de inclusión. La mayor parte de estudios concluyeron que la mayor pérdida de espacio después de la perdida prematura de un primer molar primario es en la mandíbula. Sin embargo esta pérdida es mayor cuando se pierde prematuramente un segundo molar primario.

De estos 15 artículos, 9 6, 9, 10, 11, 14, 16, 19, 22, 25 consideraron colocar mantenedor de espacio después de la pérdida prematura del primer molar primario, debido a la perdida de espacio por un mayor movimiento mesial del segundo molar primario superior y un mayor movimiento distal del canino inferior. De estos 9 solo $2^{6,16}$ consideraron que la longitud y ancho del arco dental eran afectados al perder prematuramente un primer molar inferior primario; 3 artículos $\left(\right.$ Cuoghi $^{10}$, Kumari ${ }^{9}$ y Andreeva ${ }^{19}$ ) consideraron que la mayor pérdida de espacio se da en los primeros meses después de la perdida prematura y solo el artículo de Park ${ }^{11}$ consideró colocar mantenedor de espacio antes o durante la erupción del primer molar permanente.

Los $6^{7,15,23,24,28,29}$ artículos restantes no consideraron colocar mantenedor de espacio, debido a que la pérdida de espacio que se produce es mínima y no afecta la longitud y ancho de la arcada dental; estos consideran que si bien hay un movimiento distal del canino, este es de $1 \mathrm{~mm}$, el cual no es significativo. Cabe mencionar que estos 6 artículos estudiaron solo primeros molares superiores primarios (tabla 1).

Sin embargo se considera que muchos factores condicionan a la perdida de espacio después de la perdida prematura de un primer molar primario, como por ejemplo, la cantidad de discre- pancia negativa diente-arco, el status de erupción del primer molar permanente, el momento de la perdida prematura, entre otros ${ }^{11,12,24}$.

\section{Discusión:}

Las investigaciones en relación a los cambios de espacio en el arco dental después de la pérdida prematura del primer molar primario es difícil de realizar con precisión debido que existen variables multifactoriales que influyen en los resultados.

Algunos estudios cuestionan la decisión de colocar mantenedor de espacio después de la pérdida de la primer molar primario, ya que consideran la perdida de espacio no significativa cuando se pierde este molar primario después de la erupción del primer molar permanente (entre los 7,5 - 8 años). ${ }^{11,12,13}$ Se concluye que puede no ser necesario un mantenedor, porque la perdida de espacio es marginal, y sobre todo en casos donde hay una buena interdigitación entre los segundos molares primarios y primeros molares permanentes que evitan el desplazamiento mesial de dichos molares y la pérdida de espacio. ${ }^{11}$

Northway ${ }^{14}$ observó la pérdida prematura de primeros molares primarios superiores y no encontró migración mesial aparente del primer molar permanente; lo que si se observó fue aumento de la dimensión de arco especialmente en el segmento anterior (anchura y longitud intercanina), lo que sugiere que no hay necesidad del uso de mantenedores de espacio. ${ }^{15}$

Sin embargo, hay algunos estudios que han demostrado que después de la pérdida prematura del primer molar primario, no solo se puede mesializar el segundo molar primario, sino tam- 


\begin{tabular}{|c|c|c|c|c|c|c|c|}
\hline \multirow{2}{*}{ Autor/año } & \multirow{2}{*}{ País } & \multirow{2}{*}{$\begin{array}{l}\text { Tamaño de } \\
\text { la muestra }\end{array}$} & \multirow{2}{*}{$\begin{array}{l}\text { Edad } \\
\text { (años) }\end{array}$} & \multirow{2}{*}{$\begin{array}{l}\text { Diente } \\
\text { primario }\end{array}$} & \multirow{2}{*}{$\begin{array}{l}\text { Tiempo de } \\
\text { evolución }\end{array}$} & \multicolumn{2}{|c|}{ Resultado } \\
\hline & & & & & & $\begin{array}{l}\text { Perdida } \\
\text { espacio }\end{array}$ & $\begin{array}{l}\text { Mantenedor de } \\
\text { espacio }\end{array}$ \\
\hline $\begin{array}{l}\text { Ronnerman and Thilander }{ }^{29} \\
1977\end{array}$ & Suecia & 58 & $6-7$ & $1^{\circ}$ y $2^{\circ}$ molar & $3,5,7$ años & $\begin{array}{c}(+) 2^{\circ} \text { molares } / \\
(+) 1^{\circ} \text { molar } \\
\text { inferior }\end{array}$ & $2^{\circ}$ molares \\
\hline $\begin{array}{c}\text { Northway and cols } \\
1984\end{array}$ & Canadá & 107 & 6 & $1^{\circ}$ y $2^{\circ}$ molar & 6 años & $(+) 2^{\circ}$ molares & $2^{\circ}$ molares \\
\hline $\begin{array}{c}\text { Cuoghi and cols }{ }^{10} \\
1998\end{array}$ & Brasil & 31 & $6-10$ & $1^{\circ}$ molar inferior & $\begin{array}{c}6,12,18 \\
\text { meses }\end{array}$ & $\begin{array}{l}\text { Movimiento } \\
(+) \text { distal cani- } \\
\text { no/ } 6 \text { meses }\end{array}$ & $\mathrm{Si}$ \\
\hline $\begin{array}{c}\text { Lin and Chang }{ }^{6} \\
1998\end{array}$ & China & 21 & $5-7$ & $\begin{array}{l}1^{\circ} \text { molar infe- } \\
\text { rior }\end{array}$ & 8 meses & $\begin{array}{l}\text { Movimiento } \\
(+) \text { distal cani- } \\
\text { no/longitud y } \\
\text { ancho de arco. }\end{array}$ & $\mathrm{Si}$ \\
\hline $\begin{array}{l}\text { Northway }^{14} \\
2000\end{array}$ & Canadá & 13 & 6 & $\begin{array}{l}1^{\circ} \text { molar supe- } \\
\text { rior }\end{array}$ & 6 años & $\begin{array}{l}\text { Movimiento } \\
\text { mesial de } 2^{\circ} \\
\text { molar }\end{array}$ & $\mathrm{Si}$ \\
\hline $\begin{array}{l}\text { Kumari BP and kumara } \\
\text { NR }^{9} 2006\end{array}$ & India & 30 & $6-9$ & $\begin{array}{l}1^{\circ} \text { molar infe- } \\
\text { rior }\end{array}$ & $\begin{array}{c}2,4,6,8 \\
\text { meses }\end{array}$ & $\begin{array}{l}\text { Movimiento } \\
(+) \text { distal cani- } \\
\text { no/ } 4 \text { meses }\end{array}$ & $\mathrm{Si}$ \\
\hline $\begin{array}{c}\text { Lin and cols }{ }^{23} \\
2007\end{array}$ & China & 19 & $4-7$ & $\begin{array}{l}1^{\circ} \text { molar supe- } \\
\text { rior }\end{array}$ & 6 meses & $\begin{array}{l}\text { Movimiento } \\
\text { distal canino } \\
(1 \mathrm{~mm})\end{array}$ & No \\
\hline $\begin{array}{l}\text { Park and cols }{ }^{11} \\
2009\end{array}$ & Korea & 13 & $5-10$ & $\begin{array}{l}1^{\circ} \text { molar supe- } \\
\text { rior }\end{array}$ & 12 meses & $\begin{array}{l}\text { No después } \\
\text { de } 1^{\circ} \text { molar } \\
\text { permanente }\end{array}$ & $\begin{array}{l}\text { Si (Antes/ durante } \\
\text { erupción de } 1^{\circ} \\
\text { molar permanente }\end{array}$ \\
\hline $\begin{array}{c}\text { Banderia and } \text { cols }^{28} \\
2011\end{array}$ & Brasil & 55 & $6-9$ & $1^{\circ}$ y $2^{\circ}$ molares & $\begin{array}{c}3,6,10 \\
\text { meses }\end{array}$ & $\begin{array}{c}(+) 2^{\circ} \text { molares / } \\
3 \text { meses }\end{array}$ & $2^{\circ}$ molares \\
\hline $\begin{array}{l}\text { Yai-Tin-Lin and cols }{ }^{15} \\
2011\end{array}$ & Taiwan & 13 & $5-7$ & $\begin{array}{l}1^{\circ} \text { molar supe- } \\
\text { rior }\end{array}$ & 1 año & $\begin{array}{l}\text { Movimiento } \\
\text { distal canino } \\
\qquad(1 \mathrm{~mm})\end{array}$ & No \\
\hline $\begin{array}{l}\text { Al-Dulayme } \\
2013\end{array}$ & Irak & 50 & $8-9$ & $\begin{array}{l}1^{\circ} \text { y } 2^{\circ} \text { molar } \\
\text { superior }\end{array}$ & 6 y 12 meses & $\begin{array}{l}(+) 2^{\circ} \text { molar } \\
\text { deciduo. } \\
1^{\circ} \text { molar }(+ \\
\text { mov. Distal } \\
\text { canino) }\end{array}$ & $\mathrm{Si}$ \\
\hline $\begin{array}{l}\text { Al-Dulayme D. and } \\
\text { Al-Dulayme M. }{ }^{16} \\
2014\end{array}$ & Irak & 50 & $8-9$ & $\begin{array}{l}1^{\circ} \text { y } 2^{\circ} \text { molar } \\
\text { inferior }\end{array}$ & 6,12 meses & $\begin{array}{c}1^{\circ} \text { molar ( } \\
\text { mov distal } \\
\text { canino y me- } \\
\text { sial } 1^{\circ} \text { molar } \\
\text { permanente } \\
\text { /longitud y } \\
\text { ancho de arco }\end{array}$ & $\mathrm{Si}$ \\
\hline $\begin{array}{l}\text { Alexander and cols }{ }^{25} \\
\qquad 2015\end{array}$ & EE.UU & 226 & $7-8$ & $1^{\circ}$ molar & 9 meses & $\begin{array}{l}\text { (+) mandibu- } \\
\text { la/ Patrón de } \\
\text { cara }\end{array}$ & $\mathrm{Si}$ \\
\hline $\begin{array}{l}\text { Lin Y-TJ and Lin Y-T } \\
2016\end{array}$ & Taiwan & 19 & $5-7$ & $\begin{array}{l}1^{\circ} \text { molar supe- } \\
\text { rior }\end{array}$ & 81 meses & $\begin{array}{l}\text { Mov.mesial } \\
\text { de } 1^{\circ} \text { molar } \\
\text { permanente o } \\
2^{\circ} \text { molar de- } \\
\text { ciduo minima } \\
\text { o ausente }\end{array}$ & No \\
\hline $\begin{array}{l}\text { Andreeva and cols. }{ }^{19} \\
2016\end{array}$ & Bulgaria & 90 & $6-9$ & $1^{\circ}$ y $2^{\circ}$ molares & 2,6 meses & $\begin{array}{l}(+) 2^{\circ} \text { molar } \\
\text { deciduo/ }(+) 1^{\circ} \\
\text { molar deciduo } \\
\text { inferior (mov. } \\
\text { Distal canino) }\end{array}$ & $\begin{array}{l}\text { Si ( dentro de } \\
\text { los } 2 \text { meses y } \\
\text { apiñamiento) }\end{array}$ \\
\hline
\end{tabular}

(+): Mayor prevalencia.

Tabla 1. Uso de mantenedor de espacio. 
bién se puede distalizar el canino, generando cambios a nivel del espacio por ambos lados; ${ }^{7}$ 9,16 esto se debe a la erupción del incisivo lateral permanente que ejerce una fuerza distalmente hacia el canino primario produciendo el movimiento de este. ${ }^{17}$

Además, la perdida de espacio y el desarrollo de la maloclusión son más críticos en la mandíbula que en la maxila, cuando la perdida prematura de la primer molar primario ocurre antes o durante la erupción del primer molar permanente, por lo cual se recomienda el uso de mantenedor de espacio ${ }^{5,12,18}$.

La pérdida de espacio disminuye con el tiempo, muchos autores indican que esta pérdida se da dentro de los 6 meses después de la perdida prematura, ${ }^{15,19}$ sin embargo, la mayor pérdida se da durante los 4 primeros meses. ${ }^{9}$ Es por ello que si un mantenedor de espacio no es colocado poco después de la pérdida prematura, la oportunidad de evitar la pérdida de espacio en el sitio de extracción puede haber ya pasado, ${ }^{20}$ debido a que la incidencia de la perdida de espacio va disminuyendo un poco después de los 6 meses. ${ }^{22}$

Muchos autores concluyen que la pérdida prematura del primer molar primario no genera una pérdida clínicamente significativa de ancho o perímetro del arco; ya que la pérdida de espacio significativa se observa en el sitio extracción, pero no en perímetro, anchura o longitud del arco. 9, 20, 22 Por lo tanto, una pérdida aproximada de $1,5 \mathrm{~mm}$ en un cuadrante mandibular o $1.0 \mathrm{~mm}$ en un cuadrante maxilar puede ser estadísticamente significativa, aunque no es clínicamente significativa para justificar el uso de un mantenedor de espacio. ${ }^{20,23,24}$

Por lo tanto, las decisiones relativas al uso de mantenedores de espacio deben estar guiadas por diversos factores como la edad del niño, grado de apiñamiento y maloclusión, en qué arcada se pierde el diente, el status de erupción del primer molar permanente, la etapa de formación del sucesor permanente, la interdigitación, hábitos orales y algunos consideran el potencial de crecimiento facial. ${ }^{24,} 25$

Se considera que cuanto más temprano se pierde un diente, mayor es la probabilidad de pérdida de espacio. Por otro lado, el grado de apiñamiento está directamente relacionado con la velocidad y el grado de pérdida de espacio, por lo cual habrá mayor pérdida de espacio si el apiñamiento es grave. ${ }^{26}$ Así mismo pacientes con maloclusión Clase I son menos propensos a esta pérdida de espacio ya que una buena interdigitación de los molares permanentes los hace menos susceptibles. ${ }^{27}$ Algunos estudios ${ }^{15,28}$ indican el uso de mantenedor de espacio después de la pérdida prematura del primer molar primario cuando esta pérdida es bilateral, y no cuando es unilateral ya que en este caso no se da muchos cambios y más aún cuando ya erupciónó el primer molar permanente.

Esta revisión de literatura muestra que la evidencia a favor o en contra el uso de los mantenedores de espacio después de la pérdida temprana del primer molar primario es aún muy limitada.

\section{Conclusiones}

La Pérdida prematura del primer molar primario tiene menores efectos en las dimensiones de los arcos dentales, está perdida disminuye con el tiempo y su magnitud es clínicamente cuestionable; sin embargo se ha demostrado un gran efecto en el sitio de extracción por lo cual el 
mantenedor de espacio debe ser usado lo más temprano posible teniendo en consideración todos los factores que afectan está perdida y así poder evitar el desplazamiento de los dientes adyacentes. El significado clínico de la perdida de espacio no se puede generalizar a todos los pacientes, por lo cual debe ser determinada de manera individual para cada paciente. Por último los tamaños de las muestras y metodología de algunos artículos son limitados por lo cual se necesitan estudios bien controlados para proporcionar una imagen más completa de las posibles consecuencias de la pérdida prematura del primer molar primario.

\section{Referencias bibliográficas}

1. Rao AK, Sarkar S. Changes in the arch length premature loss of deciduous molars. J Indian Soc Pedod Prev Dent 1999;17(1):28-32. 2. Leite-Cavalcanti A, Menezes SA, Granville-Garcia AF, Correia-Fontes LB. Prevalence of early loss of primary molars: Study retrospective. Acta Sci Health Sci 2008; 30: 139-43.

3. Leite-Cavalcanti A, de Alencar CR, Bezerra PK, Granville-Garcia AF. Prevalence of early loss of primary molars in school children in Campina Grande, Brazil. Pak Oral Dent J 2008; 28:113-6.

4. Pedersen J, Stensgaard K, Melsen B. Prevalence of malocclusion in relation to premature loss of primary teeth. Community Dent Oral Epidemiol 1978; 6(4): 204-9.

5. Brothwell DJ. Guidelines on the use of space maintainers following premature loss of primary teeth. J Can Dent Assoc 1997; 63(10): 753, 757-60, 764-6.

6. Lin YT, Chang LC. Space changes after premature loss of mandibular primary first molar. Int J Pediatr Dent 1998; 22(4): 211-315.

7. Northway WM, Wainright RL, Demerjian A. Effects of premature loss of deciduous molars. Angle Orthod 1984; 54(4): $295-329$.

8. Ahamed SS, Reddy VN, Krishnakumar R, Mohan MG, Sugumaran DK, Rao AP. Prevalence of early loss of primary teeth in 5-10-year-old school children in Chidambaram town. Contemp Clin Dent 2012; 3(1): 27-30.

9. Kumari BP, Kumari NR. Loss of space and changes in the dental arch after premature loss of the lower primary molar: A longitudinal study. J Indian Soc Pedod Prev Dent 2006; 24(2): 90-6.

10. Cuoghi OA, Bertoz FA, de Mendonca MR, Santos EC. Loss of space and dental arch length after the loss of the lower first primary molar: A longitudinal study. J Clin Pediatr Dent 1998; 22(2): 117-20.

11. Park K, Jung DW, Kim JY. Three-dimensional space changes after premature loss of a maxillary primary first molar. Int J Pediatr Dent 2009; 19(6): 383-89.

12. De Almeida JC, Küchler EC, Silva TK, Alves LA, Costa MC. Perda precoce de dentes decíduos: prevalência, conseqüência e tratamento. Int J Dent Recife 2011; 10: 126-30.

13. Kisling E, Hoffding J. Premature loss of primary teeth: Part V, treatment planning with due respect to the significance of drifting patterns. J Dent Child 1979; 46(4): 300-6.

14. Northway WM. The not-so-harmless maxillary primary first molar extraction. J Am Dent Assoc 2000; 131(12); 1711-20.

15. Lin YT, Lin WH, Lin YT. Twelve-month space changes after premature loss of a primary maxillary first molar. Int J Paediatr Dent 2011; 21(3): 161-6.

16. Al-Dulayme DA, Al-Khannaq MR. Mandibular dental arch dimensional changes following prematurely lost deciduous molars. J Oral Dent Res 2014; 2(1): 22-28.

17. Rapp R, Demiroz I. A new design for space maintainers replacing prematurely lost first primary molars. Pediatr Dent 1983; 5(2): $131-4$.

18. Ghafari J. Early treatment of dental arch problems. I. Space maintenance, space gaining. Quintessence Int 1986; 17(7): 423-32. 19. Andreeva RS, Arnautska HI, Belcheva AB, Georgieva MT, Dimitrov EV. Loss of space according to the time and the type of the premature extracted deciduous teeth. J of IMAB. 2016; 22(2): 1169-71. 
20. Tunison W, Flores-Mir C, ElBadrawy H, Nassar U, El-Bialy T. Dental arch space changes following premature loss of primary first molars: a systematic review. Pediatr Dent 2008;30(4):297-302.

21. Owen DG. The incidence and nature of space closure following the premature extraction of deciduous teeth: a literature study. Am J Orthod 1971; 59(1): 37-49.

22. Al-Dulayme DA. The effects of unilateral premature loss of maxillary primary molars on the dental arch dimensions. J Bagh Coll Dentistry 2013; 25(4) : 95-99.

23. Lin YT, Lin WH, Lin YT. Immediate and six-month space changes after premature loss of a primary maxillary first molar. J Am Dent Assoc 2007; 138(3): 362-8.

24. Lin Y-TJ, Lin Y-T. Long-term space changes after premature loss of a primary maxillary first molar. J Dent Sci (2016), http://dx.doi.org/10.1016/j.jds.2016.06.005

25. Alexander SA, Askari M, Lewis P. The premature loss of primary first molars: space loss to molar occlusal relationships and facial patterns. Angle Orthod 2015;85(2):218-23.

26. Laing E, Ashley P, Naini FB, Gill DS. Space maintenance. Int J Paediatr Dent 2009; 19(3): 155-62.

27. Law CS. Management of premature primary tooth loss in the child patient. J Calif Dent Assoc 2013; 41(8): 612-8.

28. Bandeira Macena MC, Tornisiello Katz CR, Heimer MV, de Oliveira e Silva JF, Costa LB. Space changes after premature loss of deciduous molars among Brazilian children. Am J Orthod Dentofacial Orthop 2011; 140(6): 771-8.

29. Ronnerman A and Thilander B. A lorigitudinal study on the effect of unilateral extraction of primary molars. Scand. J. Dent Res. 1977: 85: 362-372.

Recibido: 23/11/2016

Aceptado: 14/12/2016

Correspondencia: Elizabeth Fiorella Pino Guerrero, elizabeth.pino.g@upch.pe.

Pasaje el Sol. Condominio Iquique Block Z dpt. 101 Bellavista - 992508010 